\title{
A novel laboratory pushing the limits for optics-based basic turbulence investigations
}

\author{
S. L. Ribergaard ${ }^{1}$, Y. Zhang ${ }^{1}$, H. Abitan ${ }^{1}$, J. S. Nielsen ${ }^{1}$, N. S. Jensen ${ }^{1}$, C. M. \\ Velte $^{1 *}$, \\ ${ }^{1}$ Turbulence Research Laboratory, Technical University of Denmark, Department of Mechanical \\ Engineering, Kgs. Lyngby, Denmark \\ *cmve@dtu.dk
}

\begin{abstract}
Developments in theoretical investigations and experimental techniques are reaching a level of maturity for which it is finally becoming possible to answer some of the most pressing questions in turbulence. The prevailing classical theories all have their strengths and drawbacks based on their respective principal assumptions. To better understand the implications of these assumptions, we have developed a theoryintensive experimental strategy. For these purposes, a laboratory has been established at the Department of Mechanical Engineering, Technical University of Denmark. The objective being to provide the data necessary to test the (bounds of) validity of the existing theories; Most prominently the classical RichardsonKolmogorov-Batchelor paradigm, but also other generally adopted views such as Rapid Distortion Theory and Equilibrium Similarity. The measurements will be analyzed within a novel theoretical framework that enables not only quantification of the degree to which the small and intermediate scale turbulence behaves according to the existing theories (and their central assumptions), but also unveiling the underlying processes that create the respective state of turbulent flow. The present work will describe the current state of the developments of building up the laboratory.
\end{abstract}

\section{Introduction}

\subsection{Rationale for proposed experiments}

Our current general understanding of turbulence is not based exclusively on the equations that govern fluid flow. Central to the classical understanding is a set of assumptions ultimately based on an analogy between the fluid and the flow. Similar to molecules in a thermodynamic equilibrium in relation to a macroscopic thermodynamic state, no matter what the dynamics are at the large scales, the small and intermediate scales of turbulence are assumed to be effectively decoupled from the energy containing large scales, leading to a (universal) statistical local (small and intermediate scale) equilibrium as long as the Reynolds number is sufficiently high, see Figure 1(a). However, it is important to remember that these very fundamental assumptions are not anchored in the equations that govern fluid flow.

In a very simple thought experiment, as presented earlier in Velte and Buchhave (2021), one can imagine the process of two large co-rotating scales in close vicinity to each other. As they rotate, smaller structures will be able to form in between and energy will be directly transferred between these large and small structures. Thus, even dynamic variations in time will be able to transfer directly between these widely different sizes of structures. This thought experiment thus illustrates an intuitive example of non-local interactions. If non-local interactions can occur, then dynamics from the large scales can be transferred directly to the small scales, and the second assumption of local equilibrium is also potentially violated. So for the theory to remain valid, if this picture holds true, all scales are required to be in equilibrium. This is valid, at least in an averaged sense, in some flows - like the round turbulent jet.

Carefully designed and conducted experiments that are based on theoretical considerations is an important step in increasing our understanding. That is why we at the Turbulence Research Laboratory at the Technical University of Denmark aim to provide this solid empirical foundation to properly test local equilibrium. The measurements will be analyzed in light of the governing equations using a theoretical 


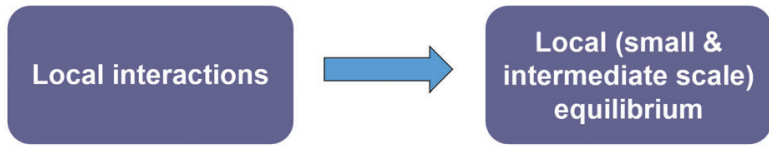

(a) Local interactions leading to local equilibrium.

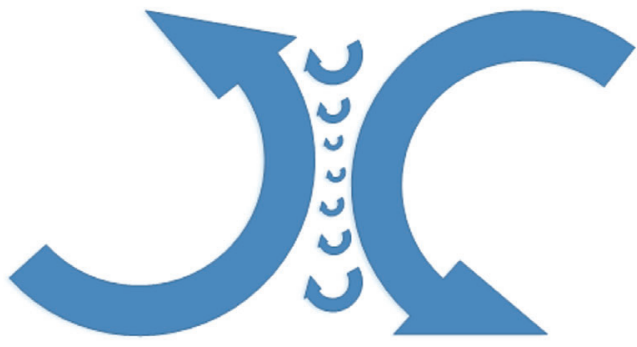

(b) Thought experiment: non-local interactions and non-equilibrium.

Figure 1: (Left) The principal assumptions of the Richardson-Kolmogorov paradigm. (Right) Thought experiment illustrating the possibility for non-local interactions.

framework carefully developed over the past years. In the current work, the objective is to describe the experimental aspects of this effort.

Previously, we have developed formalism that shows that time must be included in the analysis as an independent variable, Buchhave and Velte (2019), which has previously been neglected, c.f. Batchelor (1953). The developments are, however, limited to the description of the frequency content in a (measurement) point for statistically stationary turbulence. To be able to properly describe the underlying processes of energy exchange between wavenumber and frequency components, a four-dimensional full-field description is required. Analyzing the dynamic statistics of the turbulent kinetic energy, i.e. second order moments, requires extensive amounts of data to obtain the required statistical convergence (as compared, e.g., to first order moments such as the mean velocity). At present, Direct Numerical Simulations are unable to provide such extensive statistics, not least considering the amount of parameter variations that will be necessary to answer the underlying questions.

Fortunately, with recent developments in laser technology, LEDs, high-speed cameras and big data handling as well as particle tracking processing software, existing technology has developed to a level of maturity where these experiments are in fact feasible. And although it may still not be possible to measure the smallest relevant (dissipative) scales and the energetic and inertial scales simultaneously in the same experimental test rig, it is indeed possible to conduct two separate experiments and scale the jet in a manner that matches dimensionless numbers, such as the Reynolds number, between the experiments.

In order to test the range of validity of the universal equilibrium assumption and the assumed locality of the scale interactions, our group at the DTU Turbulence Research Laboratory are in the process of establishing a state of the art laboratory tailored for these investigations. The facilities are located at, and are a part of, the Department of Mechanical Engineering at the Technical University of Denmark and funded by the European Research Council (Starting Grant 803419) and the Poul Due Jensen (Grundfos) Foundation.

Two separate experiments have thus been designed; A High-Resolution [HR] and a Full-Field [FF] experiment to test, respectively, the hypothesis of local equilibrium (as assumed by Kolmogorov) and the locality of scale interactions (the Richardson cascade). In this manner, we will be able to test directly whether these two very fundamental assumptions are invalidated and if local interactions indeed lead to universal equilibrium - independently of the dynamics at the large scales. The experiments are thus designed to target the Akilles heel of the main assumptions that the Richardson-Kolmogorov-Batchelor paradigm is based upon.

\subsection{The round turbulent jet - the optimal test bed}

Common means to create non-equilibrium flows comprise spatially accelerated flow such as contractions, flows close to separation and fractal grids. In our current investigations, the aim is to create a temporal acceleration of a flow that is very close to equilibrium when stationary - the round turbulent jet. This flow is ideal to test departures from equilibrium caused by non-steadiness since it can create, isolate and quantify deviations from local equilibrium by accelerating the flow in time. The main advantages include:

- The jet is close to equilibrium, because the flow achieves an approximate balance between produc- 
tion and dissipation of the dissipation, i.e., $D_{\varepsilon} \approx P_{\varepsilon}$. So the stationary jet is expected to follow the equilibrium Kolmogorov theory predictions.

- The round jet was the very first flow for which the theory of Kolmogorov was supported by displaying a $-5 / 3$ range in the power spectrum Gibson (1962).

- Unlike almost all evolving flows (in time or space), the far-field jet evolves at constant $R e=U_{0} \delta_{1 / 2} / \mathrm{v}$, since the jet half-width $\delta_{1 / 2} \sim x$ and the axial velocity $U_{0} \sim 1 / x$, where $x$ is the axial distance from the jet exit. Hence, any turbulent scale (e.g. the Kolmogorov scale) is always proportional to any of the other scales (e.g. the Taylor microscale or the integral scale). This includes the two-point equations (c.f. Ewing et al. (2007); Buchhave et al. (2021); Hodzic and Velte (2021)).

- The smallest scales of interest can be made sufficiently large and slow to make direct measurements of the full velocity gradient tensor possible, and thereby also the dissipation $\varepsilon$.

- It develops an inertial subrange even at relatively low Reynolds numbers.

- It can be easily accessed optically.

- In the unsteady jet, the Reynolds number can be readily changed by adjusting the jet exit velocity, without changing the initial conditions, thereby effectively isolating the Reynolds number effects (for the same upstream profile and flow geometry).

- This is also one of few flows that evolve sufficiently rapidly to be practical for these investigation purposes on laboratory scale.

- It is a canonical flow that is well understood today from extensive measurements and theory developments and basically adheres to any scaling unless altered.

No other flow has all these necessary properties altogether. The plane wake, the only other flow that also evolves at constant Reynolds number, evolves quite slowly and is therefore often unpractical with the attainable spatial measurement (or computational) domains and without a very large and long wind tunnel.

Collecting data for various degrees of acceleration is thus expected to result in various degrees of nonequilibrium. Since the stationary jet is (very close to) equilibrium, we expect to be able to calibrate the degree of non-equilibrium to the degree of temporal acceleration. This will result in a high-quality calibrated database that can be used to map out the missing gap between the two extremes; The Kolmogorov theory and Rapid Distortion Theory.

\subsection{Theory framework}

Significant effort has been put into developing a framework that allows us to understand and analyze turbulence on a more general level, including for turbulence in flows that change with time. This includes spectral analysis and decomposition of turbulence, similarity analysis, the workings of the nonlinearities in the Navier-Stokes equation and better understanding the turbulent kinetic energy and dissipation transport equations and their processes.

\section{Flow generation facilities}

\subsection{Test-cells - confinement}

In order to confine and shield the two experiments from external disturbances, each experiment resides in one of two confined test cells, separated by a control room, see Figure 2. The test cells protect the free shear flows from external disturbances and provides an environment in which one can carefully control conditions of the flow as well as measurement technical aspects such as e.g. temperature, seeding density and seeding distribution between the jet and the ambient. The test cells are inwardly painted with matt black paint to reduce unwanted reflections.

In Hussein et al. (1994), it was established that the round turbulent jet is sensitive to generate back flow in limited confinements due to momentum conservation. To experimentally approximate a free jet, one must thus design the confinement relative to the jet exit diameter and the jet width at the downstream position at 
which one wishes to carry out the measurements. If this is not taken into consideration, one may risk the added consequence that the jet can be accelerated (squeezed) out of its near global equilibrium by the finite confinement. The cells have thus been generously dimensioned to reduce back-flow to approximate free jets to as high a degree as practically possible, following the guidelines in Hussein et al. (1994). The cells have an inner height of $4.930 \mathrm{~m}$, a depth of $4.010 \mathrm{~m}$ and a length (in the streamwise direction) of $4.600 \mathrm{~m}$ (Full-Field) and 6.970m (High-Resolution), respectively. Test cells are thus designed such that the cross sectional area at the downstream position where we would like to measure, for each respective experiment, adheres to the following requirements:

- HR: At 30 jet exit diameters downstream of the jet exit, the momentum loss is less than $95 \%$

- FF: At 38 jet exit diameters downstream of the jet exit, the momentum loss is less than $1 \%$

Each test cell is equipped with four Phantom v2640 cameras (288 GB) to be used for Particle Tracking Velocimetry (PTV) and Particle Image Velocimetry (PIV) measurements. A Q-switched water cooled $40 \mathrm{~W}$ Blizz laser (Innolas Photonic $\mathrm{GmbH}$ ) with max repetition rates of $400 \mathrm{kHz}, M 2<1.4$ TEM00 at $532 \mathrm{~nm}$ will serve as the light source in the High-Resolution experiment, while the light source for the Full-Field experiment is yet to be confirmed. At present, commercially available LEDs are being employed (LaVision LED-Flashlight 300 White). However, we are currently developing our own in-house high power LED sources in several configurations for illumination of large volumes as well as exploring several additional options.

In addition, we have established a ventilation system (similar to a wind tunnel) for recirculating the air in each respective test cell and provide air from the respective test cell with the appropriate seeding density to avoid conditional seeding. The jet generator is co-axial, allowing further variations of the inlet conditions. To create temporal variations, a jet modulation mechanism has been designed and constructed. Both experiments have been prepared for full automatic control including monitoring signals of temperature variations, pressure ambient and across the jet contraction, jet modulation phase position and several other important parameters. The big data handling poses significant challenges, in that each measurement will require up to $288 \mathrm{~GB}$ of storage per camera, including handling bottlenecks for data transfer speeds. We have managed to reduce these bottlenecks so that the most time consuming aspect is the actual processing of the measurement images. All of these aspects will be described in further detail in the following.

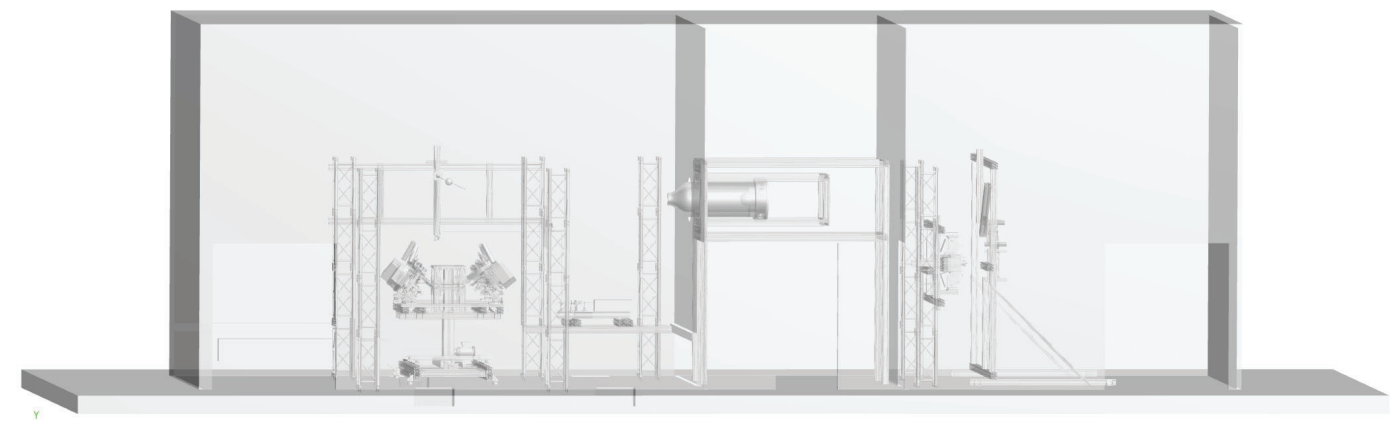

Figure 2: Illustration of the test cells constituting the flow confinement. The HR experiment is conducted in the left cell and the FF experiment in the right cell. Between the two test cells, a control room is located.

\subsection{High-Resolution experiment}

A drawing of the complete experimental setup can be found in Figure 3(a). To measure the dissipation (and even the dissipation of the dissipation), a round jet is designed large enough to produce optically and temporally resolvable scales ( $k \eta \approx 4$, where $k$ is the wavenumber and $\eta$ the Kolmogorov microscale) within the Reynolds numbers range defined $(R e \approx 2.000-75.000)$. Using particle tracking techniques, the full velocity gradient tensor will be measured instantaneously. PTV algorithms have today reached a level of maturity where even spatial velocity gradients can be measured accurately. The 'Shake the box' algorithm is particularly well suited for this, c.f. Schanz et al. (2016).

The practical limitations such as the resolution requirements in combination with the required size of the jet (and consequently the jet confinement), ultimately results in a magnification close to $\sim 1: 1$. Light 


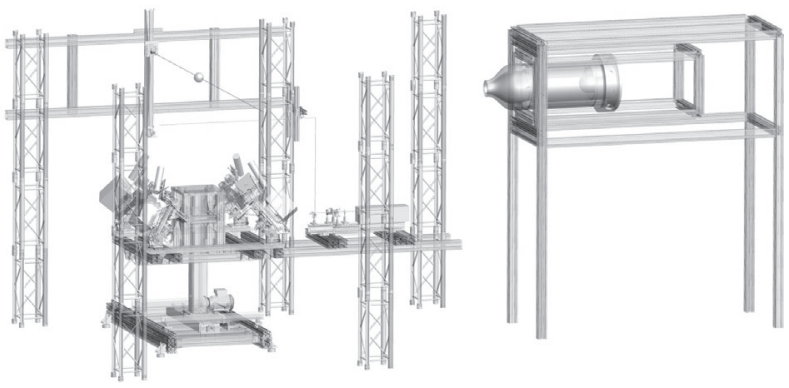

(a) Complete HR setup

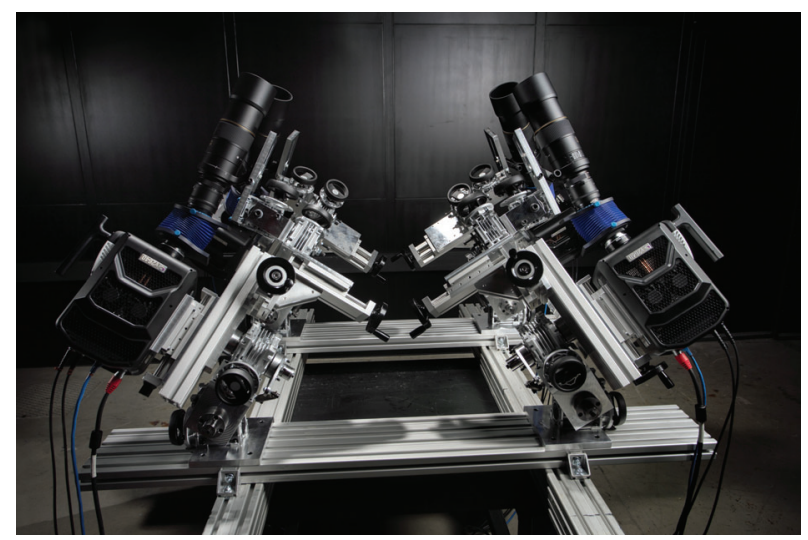

(b) Camera setup (preliminary)

Figure 3: (Left) Drawing showing the complete HR setup. (Right) Picture of a preliminary version of the camera setup used for dissipation measurements.

budget considerations are important in these experiments, but proper imaging is still proven to be possible. Another challenge with the high magnification arises in terms of the resulting large Scheimpflug angles required. Since such Scheimpflug-adaptors are not commercially available (and are not designed to carry the heavy optics), we have developed a solution tailored for this purpose with seven degrees of freedom which is described in a companion paper, see Ribergaard et al. (2021). See also Figure 3(b) for a picture of a preliminary version of the camera setup. The Scheimpflug adaptor is furthermore able to establish Scheimpflug focusing along one or two axes, that provides more general possibilities for combining positioning and alignment compared to commonly available or traditional Scheimpflug mounts.

To be able to accurately trace the flow at the resolved temporal and spatial scales, $\sim 1 \mu \mathrm{m}$ seeding particles consisting of atomized oil drops will be used. Preliminary light budget estimates as well as direct tests have confirmed that the particles can be properly imaged at the required scales with the existing equipment. Extensive experience of the team with using these seeding tracers indicates that no significant problems with loss of seeding particles is expected within the recirculation system and hence the seeding of the jet and ambient can be considered homogeneous to a good approximation.

\subsection{Full-Field experiment}

Experimentally testing the degree of locality of the interactions between wavenumber components requires measurements that to some degree cover the global dynamics of the flow. This includes all three spatial dimensions and time, as omitted dimensions will be aliased onto the measured ones and thus not produce a complete and true representation of the actual flow.

In the past decade, developments in volumetric PTV techniques facilitated particle tracking in relatively large volumes (above $10^{3} \mathrm{~cm}^{3}$ ) compared to what was previously possible. This was demonstrated by using $300 \mu \mathrm{m}$ diameter helium filled soap bubbles and the 'Shake the Box' algorithm (Schanz et al. (2016)) at $\mathrm{kHz}$ repetition rates, c.f. Barros et al. (2021).

Although the helium-filled soap bubbles are sufficiently large to be measured across such large domains, the commercially available $\sim 15 \mu \mathrm{m}$ air-filled tracer soap bubbles of TSI exhibit critical properties motivating us to instead employ these in our experimental investigations. Some of the necessary properties of these smaller bubble traces include; A high degree of durability to keep the seeding density of the jet flow close to that of the ambient flow of the jet (including passing through contractions, screens, pumps etc.), an ability to accurately trace the flow at the scales resolved in the measurements and a high attainable seeding density so the bubbles can be uniformly and sufficiently densely dispersed in the test cell and recirculation system. Furthermore, our measurements require millimeter accuracy of the PTV measurement in large volumes, which favors using $15 \mu \mathrm{m}$ soap bubbles in the current experiments. Since Mie-Scattering is proportional to the quadratic power of the particle diameter, it is found that the required pulse energy for such large volumes is about 400 times larger than that required for the $300 \mu \mathrm{m}$ bubbles (Zhang et al. (2021); Abitan et al. (2021)). This corresponds to lasers with pulse energy and average power that are not available commercially and their development would be prohibitively expensive. 


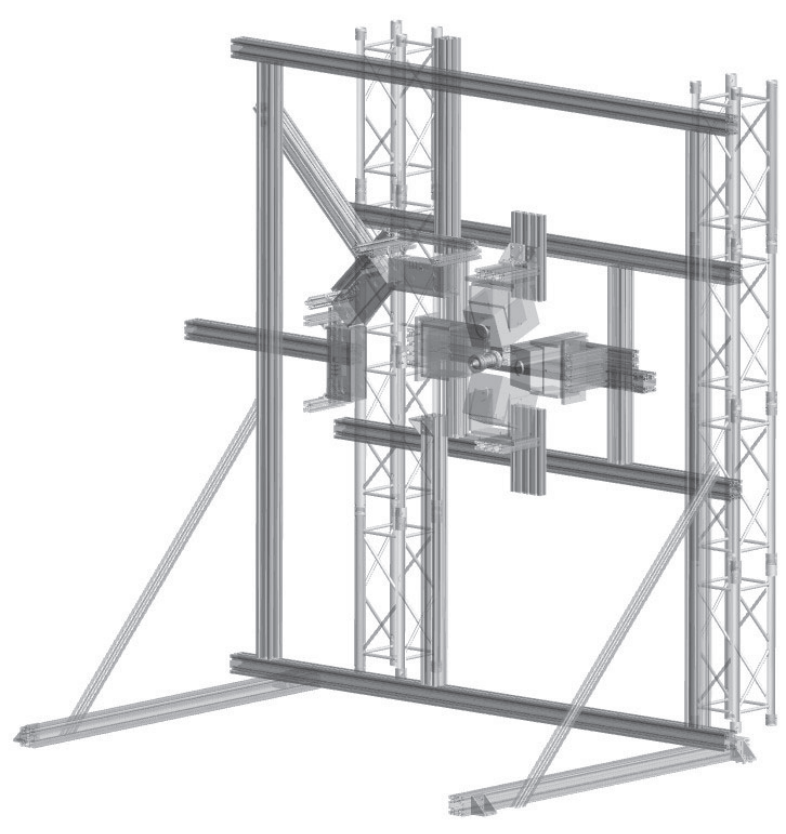

(a) Drawing of the complete FF setup (preliminary)

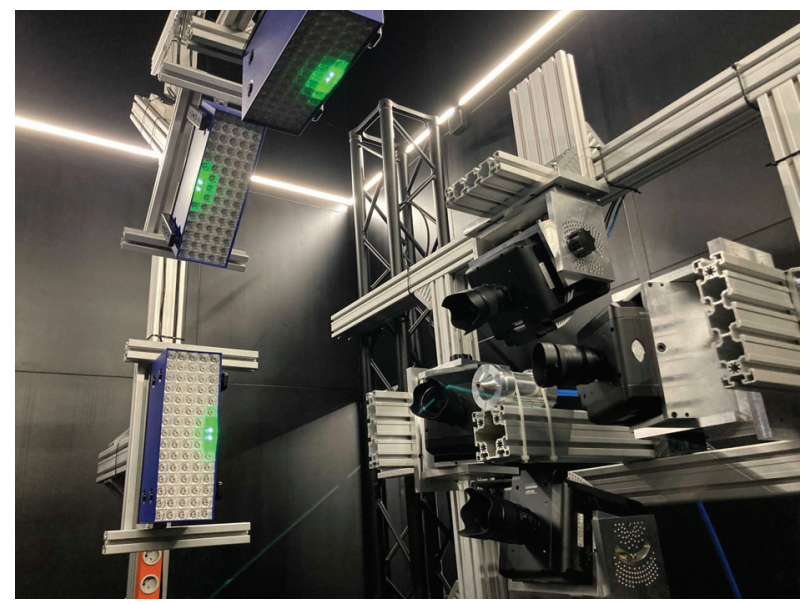

(b) Picture of the complete FF setup (preliminary)

Figure 4: (Left) Drawing showing the complete FF setup. (Right) Picture of the current preliminary version of the camera, LED and jet setup used for global jet measurements in four-dimensions.

As a consequence, between the two bubble seeding types there exists a gap in previously attained measurement volume sizes for high-speed imaging due to restrictions in light budget, see Barros et al. (2021). The current measurements exist within this gap (Zhang et al. (2021)). To be able to extend the Field of View using the $\sim 15 \mu \mathrm{m}$ air-filled soap bubbles, several techniques have be tested and the most promising one(s) will be further developed and refined - all balanced in relation to the resolved turbulent spatial and temporal scales as described in the companion papers Zhang et al. (2021) and Abitan et al. (2021). The techniques for large Volume PTV illumination explored comprise:

- Laser light sheet scanning using an acousto-optic modulator for fast velocity light sheet scanning in combination with an off-axis parabolic mirror yielding a volumetric and parallel beam scanning, see Abitan et al. (2021).

- Multi reflections between two opposing parallel mirrors to maximize the usage of the available light, see Abitan et al. (2021).

- Commercially available LEDs (LaVision LED-Flashlight 300 White).

- In-house development LEDs tailored for the FF experiment (under development).

At present, we are using the commercial LEDs, see Figures 4(a) and 4(b). But the most targeted effort to address this challenge constitutes developing our own in-house high power LED sources in several configurations for illumination of large volumes that are seeded with $15 \mu \mathrm{m}$ soap bubbles. We develop robust electronic drivers that are suitable for driving high power LEDs (such as those offered by Luminous). We address the heat management so that the LEDs will be easy to use in experiments and we investigate optics to collimate the high power LEDs in the most efficient manner. Further, we are still exploring the first two options for risk mitigation.

\subsection{Jet Flow Generator \& Jet Modulation}

The axisymmetric jet flow generator, see Figure 4(a), is of a co-axial type where each nozzle can be run independently with either a chosen constant jet exit velocity or with a modulation as a zero net mass flux 


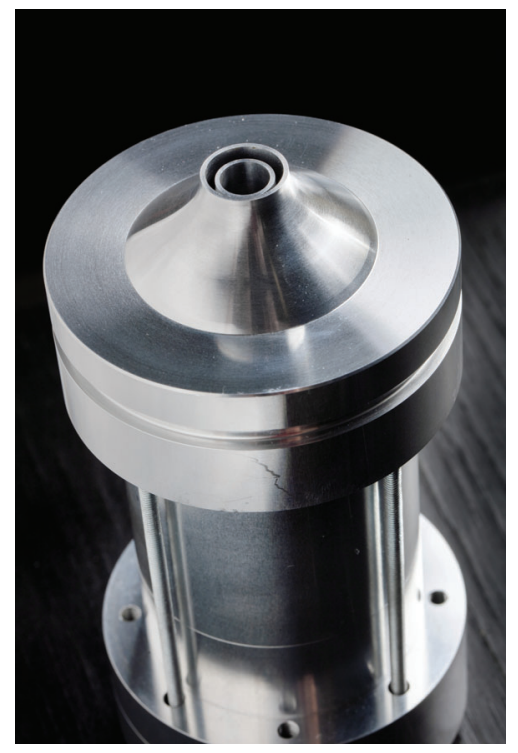

(a) Co-axial jet nozzle

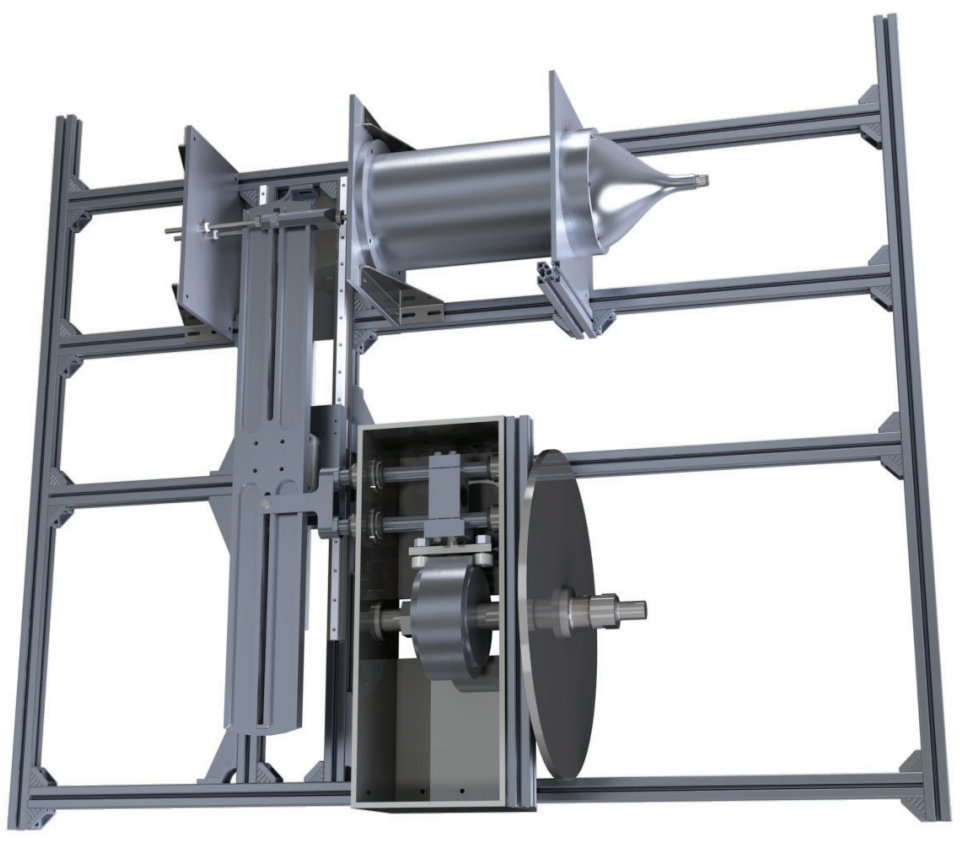

(b) Jet modulation mechanism

Figure 5: (Left) Co-axial jet nozzle for the FF experiment. (Right) Current version of jet modulation mechanism.

jet. It has been designed with a constant area ratio between the inner and the outer nozzle based on a series of RANS-simulations. The inner nozzle follows a 5 th order polynomial contraction to create a top-hat profile in accordance with the nozzle type commonly used by the group, Daehan (2001). The outer nozzle consequently has a much steeper slope. The RANS simulations indicate that no separation occurs as the walls continually contract, which has been confirmed in the final jet design. The inner and outer jets can be connected to a constant volume flow or a zero net mass flux velocity modulation, respectively.

The modulation mechanism, see Figure 4(b), is designed to create a zero net mass flux modulation that can be coupled to either the outer or the inner jet, respectively. A cylindrical cam mechanism connected to a rocker arm drives a piston moving within a cylinder to displace the air. The rocker arm has an adjustable fulcrum, allowing variable amplifications of the linear motion produced by the cam. The design further allows the generation of a general type of periodic motion by using a cam tailored to the modulation characteristics desired.

Since the modulation mechanism will be subjected to heavy dynamic loads, the reciprocal moving parts have been carefully designed and dimensioned to limit the weight while retaining the strength of the construction. The modulation mechanism can be scaled to fit either of the two experimental setups. The modulation mechanism has been designed so that it, to as large a degree as possible, creates a flow that accelerates faster than the estimated eddy turnover time. However, since all theoretical considerations are in one way or another limited to equilibrium turbulence, the actual required accelerations will have to be tested and confirmed in the experiments.

\subsection{Air Recirculation}

The air recirculation operates with two parallel wind tunnel recirculation circuits, each lining emanating into one of the two respective test cells, see Figure 6. The two test cells have a similar design, except for the seeding and general dimensioning according to each respective volume flow. The inner and outer jets are furthermore connected to independent air recirculation systems, consisting of blowers, valves, filters, flow meters and pressure sensors. The difference between the two circuits is that the inner jet circulation has three valves to be able to alternate between injecting a steady flow of air into the inner jet directly or to replace the air circulation with the output of the jet modulation mechanism.

Each ventilation circuit has a flow rate capacity of $1663 \mathrm{~m}^{3} / \mathrm{h}$, corresponding to a Reynolds number 
of 100.000 for each test cell, respectively. If both ventilation circuits are used simultaneously, e.g. for filtering out the bubble from the test cell, it is estimated to take approximately five minutes to exchange or filter out the air in the test cell. The air recirculation is controlled by the central automatic control system, which communicates with a LaVision acquisition system, as well as other components, to realize automatic recording and storage.

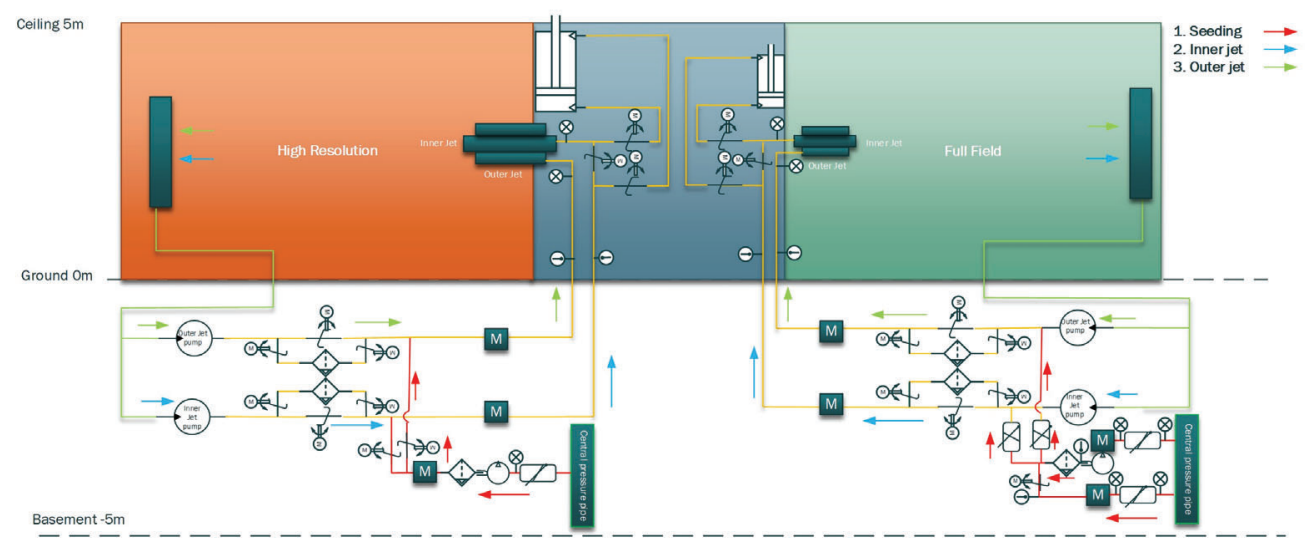

Figure 6: Schematic of the air recirculation system of both test cells, spanning between the ground level and the laboratory basement.

\subsection{Control System}

During active experiments, for security reasons and to not disturb the ongoing experiments, no one is allowed to reside inside the test cells. Hence, operation and monitoring must take place from outside of the test cells. The two test cells therefore share a common control system that consists of a management layer, a control layer and a device layer, see Figure 7. The management layer primarily provides the experiment execution plan management service, data storage and data access service. One can use the experiment execution plan management service to program the execution of the experimental plan, and use the data access service to read the data resulting from the experiment. The operator can operate the facility and monitor the status of the experiment execution process according to the experimental plan.

The facility will thus be running automatically and can be supervised by a web interface, which provides real-time process data and an emergency stop functionality. Three different emergency functionalities are provided with different purposes: (1) A quick stop for device fault, (2) a quick fresh air and bubble filtering response in case of unexpected door opening and (3) cut-off of all power in case the fire alarm is activated.

Once the experimental execution plan has been defined, the operator can start the experiment execution and check the status of all devices. The local control panel and bus connection to the central controller can be accessed through touch screen. The PIV/PTV acquisition is based on a system from LaVision.

A quality criterion for the flow and ambient conditions of the experiment can be defined, including ambient pressure and temperature, test cell pressure and temperature, air recirculation pressure and flow rate, particle density and velocity etc.. After running steadily for a fixed amount of time, e.g. an hour, the cameras and light source(s) will receive a command to begin the recording. For experiments requiring time averaging or phase averaging, the flow generation will typically continue after recording. Therefore the PIV/PTV acquisition system will continue to record after storing the images in the server, as the controller keeps the running status stable. This procedure makes data acquisition of extensive statistics, in particular for non-steady turbulent flow, more practical and manageable.

A database will be automatically generated for each experimental execution. This will contain the real-time process data (pressure, temperature, flow rate, velocity etc.) and the PIV/PTV recordings. All the data are tagged by an acquisition number, time and recording name, so it is convenient to navigate in the history of recordings and related process data. The web service of the control system will provide an online monitoring service for all computers and mobile devices with user name and password. Once the experimental measurement plan has been initiated, the experiments will run automatically. 


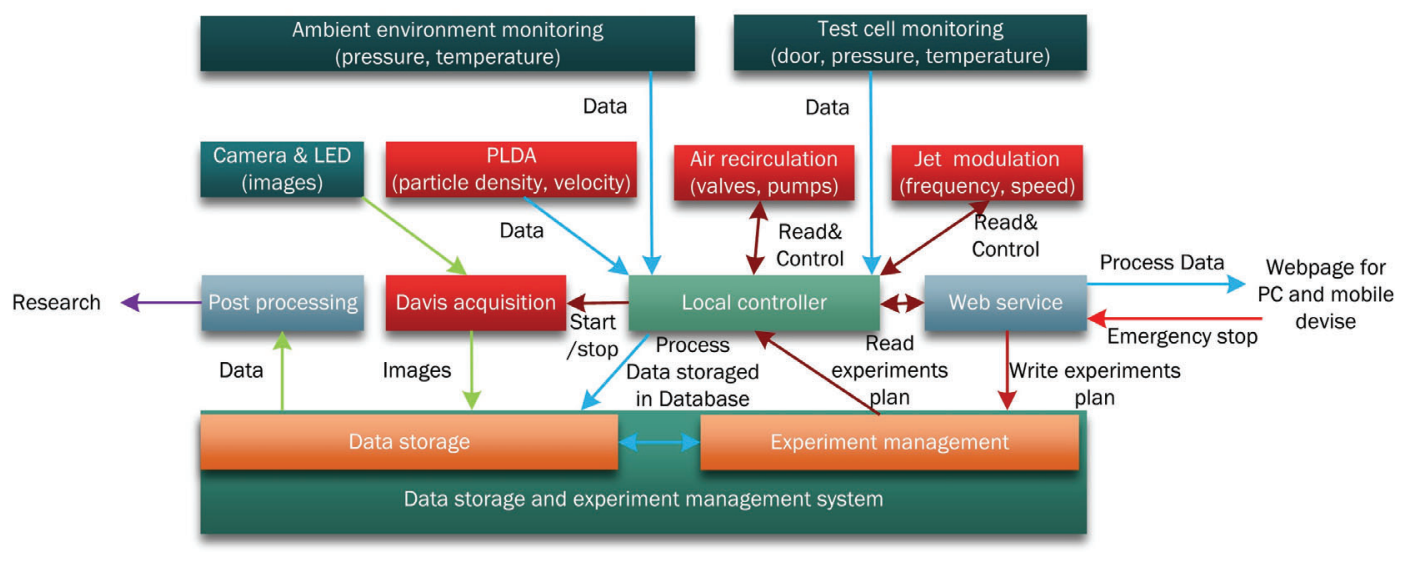

Figure 7: The control system consists of a management layer (bottom), a control layer (middle) and a device layer (top).

\subsection{Big Data Handling}

In these experiments, significant amounts of data will be produced. The data has to be handled, processed and analyzed and all at very high speeds. The setup consists of a total of eight high-speed cameras, each producing up to $288 \mathrm{~GB}$ of video data per recording, which can take as little as 5 seconds. Our infrastructure must be able to offload this data within a reasonable time-frame to a storage such that consecutive measurements can be done with minimal wait time. To store the data, a high-performance Huawei 2.5 PB network attached storage server has been acquired, connected to the two acquisition PCs via two redundant $40 \mathrm{GbE}$ fiber connections. Each acquisition PC has four $10 \mathrm{GbE}$ connections to the cameras and is responsible for generating the files from the data streams that the cameras deliver. The data handling structure is illustrated in Figure 8. When the data is stored, dedicated processing PCs will access the video files and produce the velocity field data that is then ready for our analysis. The processing will be the bottleneck limiting the data we can put through our pipeline. The final number of processing PCs needed is currently unknown.

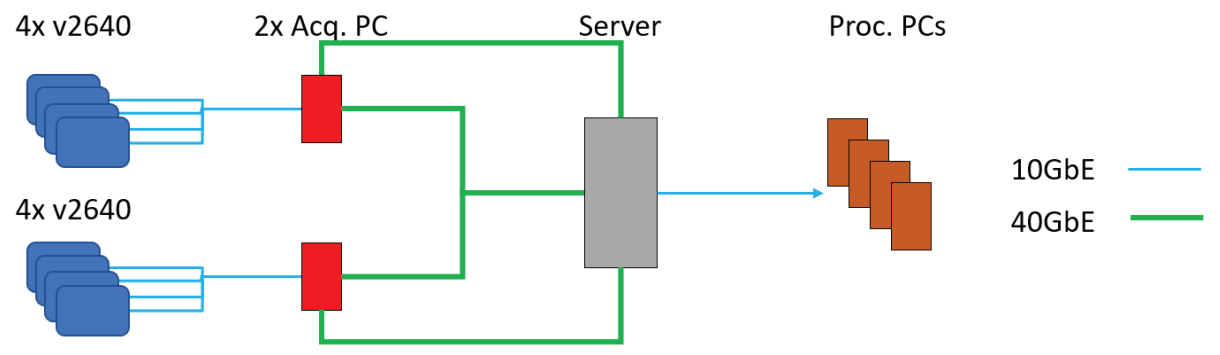

Figure 8: Big data handling structure.

\section{Conclusions}

A novel laboratory for fundamental turbulence research is in the process of being established at the Department of Mechanical Engineering at the Technical University of Denmark as a part of the DTU Turbulence Research Laboratory. The objective is to test the range of validity of classical theories, including the Richardson-Kolmogorov-Batchelor paradigm of turbulence as well as other views such as Rapid Distortion Theory and Equilibrium Similarity. The experiments are designed to push the limits for the underlying assumptions, not least the principal assumption of local interactions in wavenumber space, assumed to result in universal equilibrium in the intermediate and small scales.

The degree of equilibrium will be quantified for varying Reynolds numbers and accelerations by direct measurements of the full dissipation rate tensor. This will also provide valuable information about the 
true nature (e.g. intermittency) of the dissipation. Similarly, a matching, but smaller, jet will be used to reveal which processes lead to the resulting dissipation measured in the first experimental setup. Do local interactions lead to the final value of dissipation? Or are other kinds of processes responsible for the spectral energy flux than the classical Richardson cascade?

The experimental test cells will be fully operational in 2021.

\section{Acknowledgements}

This project has received funding from the European Research Council (ERC) under the European Unions Horizon 2020 research and innovation program (grant agreement No 803419). Financial support from the Poul Due Jensen Foundation (Grundfos Foundation) for this research is gratefully acknowledged. The Department of Mechanical Engineering at the Technical University of Denmark is also acknowledged for their generous additional support in establishing the laboratory. Professor William K. George is acknowledged for many helpful discussions. Asbjørn Dyre Jespersen and Malthe Lundsgaard are acknowledged for their contribution in developing the jet modulation mechanisms.

\section{References}

Abitan H, Zhang Y, Ribergaard SL, and Velte CM (2021) Development of optical techniques for large volume PTV measurements. in 14th International Symposium on Particle Image Velocimetry - ISPIV21, (Virtual) Chicago, Illinois, USA, August 1-4

Barros DC, Duan Y, Troolin DR, and Longmire EK (2021) Air-filled soap bubbles for volumetric velocity measurements. Experiments in Fluids 36:933-947

Batchelor GK (1953) Homogeneous Turbulence (2nd ed.). Cambridge University Press

Buchhave P and Velte CM (2019) Dynamic triad interactions and evolving turbulence spectra. Under review, arXiv:190604756 [physicsflu-dyn]

Buchhave P, Zhu C, and Velte C (2021) Similarity scaling of a free, round jet in air. in R Örlü, A Talamelli, J Peinke, and M Oberlack, editors, Progress in Turbulence IX. Springer Proceedings in Physics. Springer. iTi Conference in Turbulence ; Conference date: 25-02-2021 Through 26-02-2021

Daehan J (2001) An Investigation of the Reynolds-Number Dependence of the Axisymmetric Jet Mixing Layer Using a 138 Hot-Wire Probe and the POD. PhD dissertation, State University of New York

Ewing D, Frohnapfel B, George W, Pedersen J, and Westerweel J (2007) Two-point similarity in the round jet. Journal of Fluid Mechanics 577:309-330

Gibson MM (1962) Spectra of turbulence at high reynolds number. Nature 195:1281-1283

Hodzic A and Velte C (2021) Two-point similarity in the round jet revisited. Journal of Fluid Mechanics

Hussein HJ, Capp SP, and George WK (1994) Velocity measurements in a high-reynolds-number, momentum-conserving, axisymmetric, turbulent jet. Journal of Fluid Mechanics 258:31-75

Ribergaard SL, Olesen PJ, Jensen NS, Nielsen JS, and Velte CM (2021) Mounting and support for pseudo biaxial Scheimpflug focusing for unity-magnification, high-speed particle velocimetry. in 14th International Symposium on Particle Image Velocimetry - ISPIV21, (Virtual) Chicago, Illinois, USA, August 1-4

Schanz D, Gesemann S, and Schröder A (2016) Shake-the-box: Lagrangian particle tracking at high particle image densities. Experiments in Fluids

Velte C and Buchhave P (2021) Dynamic triad interactions and non-equilibrium turbulence. in R Örlü, A Talamelli, J Peinke, and M Oberlack, editors, Progress in Turbulence IX. Springer Proceedings in Physics. Springer. iTi Conference in Turbulence ; Conference date: 25-02-2021 Through 26-02-2021

Zhang Y, Abitan H, Ribergaard SL, and Velte CM (2021) A novel volumetric velocity measurement method for small seeding tracers in large volumes. in 14th International Symposium on Particle Image Velocimetry - ISPIV21, (Virtual) Chicago, Illinois, USA, August 1-4 